\title{
Microscopies synchrotron à SOLEIL
}

\author{
Rachid BELKHOU, Bertrand CINQUIN, Frédéric JAMME, Nicolas JAOUEN, Kadda MEDJOUBI, \\ Isabelle QUINKAL, Delphine VANTELON, Matthieu RÉFRÉGIERS \\ Synchrotron SOLEIL, L'Orme des Merisiers, 91190 Gif-sur-Yvette \\ maithieu.refregiers@synchrotron-soleil.fr
}

Le rayonnement synchrotron est un continuum d'énergie des infrarouges aux rayons X-durs. Avec l'amélioration des sources - une meilleure stabilité, une plus grande brillance, une plus faible divergence du faisceau et une meilleure cohérence - de plus en plus de lignes de lumière à travers le monde, et à SOLEIL en particulier, proposent des stations expérimentales dédiées à la microscopie.

$\mathrm{L}^{2}$ a majorité de ces lignes utilisent la grande accordabilité du rayonnement synchrotron pour accéder à une spécificité chimique directe, proposant ainsi des méthodes de microscopie sans marqueurs. Les molécules ou les atomes de l'échantillon pourront être excités directement sans intermédiaires.

On distinguera deux grands types de microscopies synchrotron :

- Des microscopies pour lesquelles la focalisation extrême du faisceau synchrotron permet d'atteindre des résolutions nanométriques. La taille du faisceau définit alors la résolution spatiale. Le faisceau est focalisé à l'aide d'optiques adaptées : diffractives, réfractives ou réflectives. Dans cette approche, l'échantillon est en général balayé au point focal du faisceau pour reconstruire une image bidimensionnelle de la zone scannée à partir des photons transmis ou émis par l'échantillon.

- Des microscopies plein champ où le faisceau n'est pas focalisé mais au contraire éclaire uniformément la zone d'intérêt dans l'échantillon. Les photons ou électrons émis sont récoltés à l'aide des détecteurs bidimensionnels. Dans ce cas, la résolution spatiale dépend principalement du système de détection. Toutes ces méthodes permettent d'utiliser des spécificités du rayonnement synchrotron telles que la cohérence, la polarisation variable, le caractère pulsé pour accéder aux propriétés de l'échantillon.

\section{Le domaine d'énergie détermine le type d'informations obtenues}

En infrarouge, les vibrations moléculaires sondées donnent des informations sur les groupes fonctionnels présents et les modifications subies par l'environnement d'une molécule. Ainsi en biologie, il est possible d'imager directement les groupes lipidiques dans les tissus sans marquage. En physico-chimie des matériaux, on peut suivre une espèce moléculaire greffée sur une surface en fonction de paramètres externes tels que la température ou la pression.

En ultraviolet lointain, de nombreux groupes aromatiques (molécules cycliques) vont naturellement fluorescer, les oxydes métalliques vont également émettre de la lumière, dont la longueur d'onde dépend de la largeur de bande interdite de leurs électrons. La pharmacocinétique de certains médicaments devient donc accessible sans marqueurs extrinsèques.

En augmentant l'énergie des photons utilisés à l'excitation, ce sont les couches électroniques des atomes qui vont être sondées. Les rayons $X$ de faible énergie, dits « mous » seront absorbés par les éléments chimiques légers. Ils offrent un avantage supplémentaire dû à une fenêtre énergétique particulière appelée fenêtre de l'eau. Entre 280 et 560 eV, l'oxygène sera moins absorbant que le carbone, pourtant plus léger que lui, et des échantillons riches en matière organique (spécimens biologiques principalement) pourront être ainsi imagés en restant hydratés. De plus, ce domaine d'énergie donne accès aux métaux de transitions.

Lors de l'absorption des rayons $\mathrm{X}$, des électrons peuvent être émis. Les photo-électrons, s'ils sont issus d'une excitation des niveaux de cœur, permettent de sonder localement une nouvelle dimension, l'énergie cinétique des électrons photoémis (X-PEEM).

Les rayons $X$ de faibles énergies dits mous $(<1 \mathrm{keV})$ ou tendres $(<3 \mathrm{keV})$ seront très fortement absorbés par les matériaux, ce qui les rend particulièrement adaptés à l'étude de matériaux faiblement denses (matière molle, matière organique...). En outre, cette gamme d'énergie présente une très grande densité de seuils d'absorption, permettant ainsi d'étudier sur la même ligne et dans les mêmes conditions les différents éléments chimiques constituants les échantillons (éléments légers, métaux de transition, alcalins, terres rares...). Il faut aussi noter que c'est dans ce domaine d'énergie que l'on obtient les meilleures résolutions spatiales $(11 \mathrm{~nm}$ en microscopie à balayage). Enfin, cette gamme d'énergie présente une particularité unique due à la fenêtre de l'eau comme indiqué plus haut. Au sein de cette gamme d'énergie les rayons $X$ sont très peu absorbés par l'eau et de ce fait elle permet d'étudier des échantillons en milieu hydraté. D'autre part, cette fenêtre contient les seuils d'absorption des atomes les plus répandus dans la matière 
organique (carbone, oxygène et azote) et par conséquent elle est particulièrement adaptée pour l'étude du Vivant en conditions les plus proches possibles de l'état natif.

À plus haute énergie, les rayons $X$ sont plus faiblement atténués. Ceci est mis à profit pour étudier de façon non-destructive des échantillons épais ou des interfaces enterrées. Le fort rendement de fluorescence $X$ dans cette gamme d'énergie la rend particulièrement adaptée à la détection d'éléments-traces.

Le rayonnement synchrotron émis par un onduleur est hautement cohérent. Une microscopie sans lentilles utilisant la cohérence des faisceaux $X$ tendres ou durs, la ptychographie, devient ainsi réalisable. La reconstruction de phase s'effectue à partir de figures de diffraction.

\section{De nouveaux contrastes pour de nouvelles imageries}

Outre le contraste d'absorption et le contraste chimique, d'autres types de contraste peuvent être obtenus en utilisant les propriétés spécifiques du rayonnement synchrotron. Ainsi, la polarisation variable permet de remonter de façon générale aux anisotropies configurationnelles, structurelles et électroniques. En particulier, I'utilisation de la polarisation circulaire permet de sonder directement l'orientation des moments magnétiques. L'utilisation combinée de la polarisation circulaire et de la microscopie $X$ offre ainsi un outil unique permettant d'imager à haute résolution la structure des domaines magnétiques dans des dissipatifs magnétiques comme les valves de spin, les jonctions tunnel...

Le caractère pulsé du rayonnement synchrotron peut être mis à contribution notamment via la mise en œuvre d'approches de type pompes-sondes, permettant de suivre des phénomènes dynamiques à l'échelle de quelques dizaines de ps et de $\mathrm{nm}$, ouvrant ainsi la voie à la caractérisation operando de systèmes dynamiques.

L'interférométrie à réseau couple l'absorption traditionnelle des rayons $\mathrm{X}$ avec la mesure du changement de phase des photons en fonction de la densité des pixels traversés dans l'échantillon (la différence de densité peut être de $0,5 \mathrm{mg} / \mathrm{cm}^{3}$ ).

\section{L'environnement}

\section{échantillon et le domaine d'énergie déterminent le domaine d'application}

La plupart des techniques synchrotron nécessitent et ont adapté des environnements échantillons spécifiques : contrôle de température, de pression, d'atmosphère. Les nécessités expérimentales vont souvent déterminer les domaines d'application d'une technique. Ainsi, les méthodes de type électronique comme la microscopie X-PEEM opèrent en général sous ultra-vide. La faible profondeur d'analyse des photoélectrons rend en outre cette technique particulièrement sensible aux premières couches atomiques de l'échantillon. Cette méthode est donc parfaitement adaptée à l'étude des surfaces et interfaces. Les $\mathrm{X}$-durs très pénétrants permettent une tomographie haute résolution des os, des argiles. Les $\mathrm{X}$-mous trouvent toutes leurs applications pour imager les matériaux magnétiques mais possèdent également un grand potentiel en sciences du vivant. Les microscopies ultraviolet et infrarouge seront particulièrement adaptées à la biologie et au Patrimoine.

\section{Quelques applications marquantes}

\section{Le vernis des Stradivarius}

Stradivarius employait des matériaux de base courants à son époque. Grâce, en particulier, aux analyses effectuées en microscopie infrarouge sur la ligne SMIS, il a été possible de montrer que, sur tous les instruments étudiés, il a appliqué un vernis constitué de deux très fines couches. La première couche, simplement à base d'huile, similaire à celle utilisée par les artistes-peintres, offre une légère pénétration du bois de l'instrument. La seconde couche est un mélange d'huile et de résine

\section{ANALYSEUR DE SPECTRE OPTIQUE OSA20}

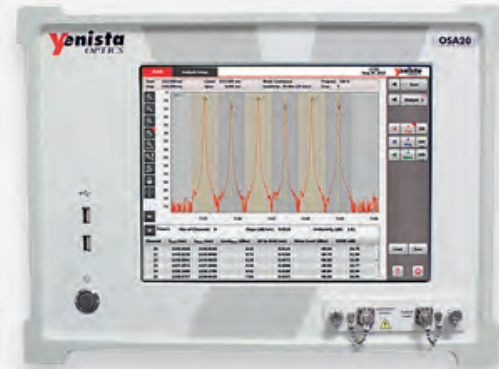

Caractéristiques clés

- Plage : 1250 - $1700 \mathrm{~nm}$

- Résolution spectrale : 20 pm

- Vitesse max : $2000 \mathrm{~nm} / \mathrm{s}$

- Précision $\lambda$ :

\pm 15 pm / $1500-1640 \mathrm{~nm}$ $\pm 25 \mathrm{pm} / 1250-1700 \mathrm{~nm}$

- Ecran tactile multipoint

- Interfaces externes :

- USB2 et USB3

- Ethernet

- Trigger IN \& OUT - GPIB

- Modes d'utilisation par application et librairie de fonctions d'analyse

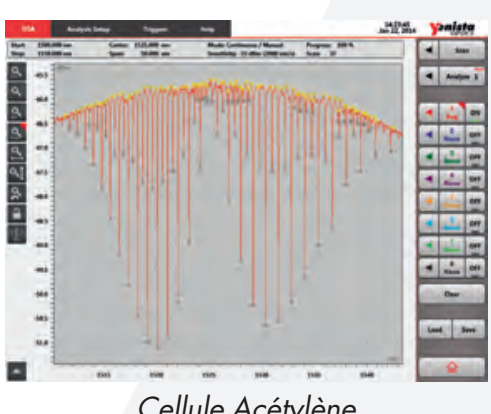

Cellule Acétylèn

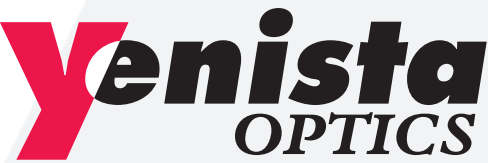

$$
\begin{gathered}
\text { Tél. : +33 (0)29648 } 3716 \\
\text { sales-emea@yenista.com } \\
\text { www.yenista.com }
\end{gathered}
$$



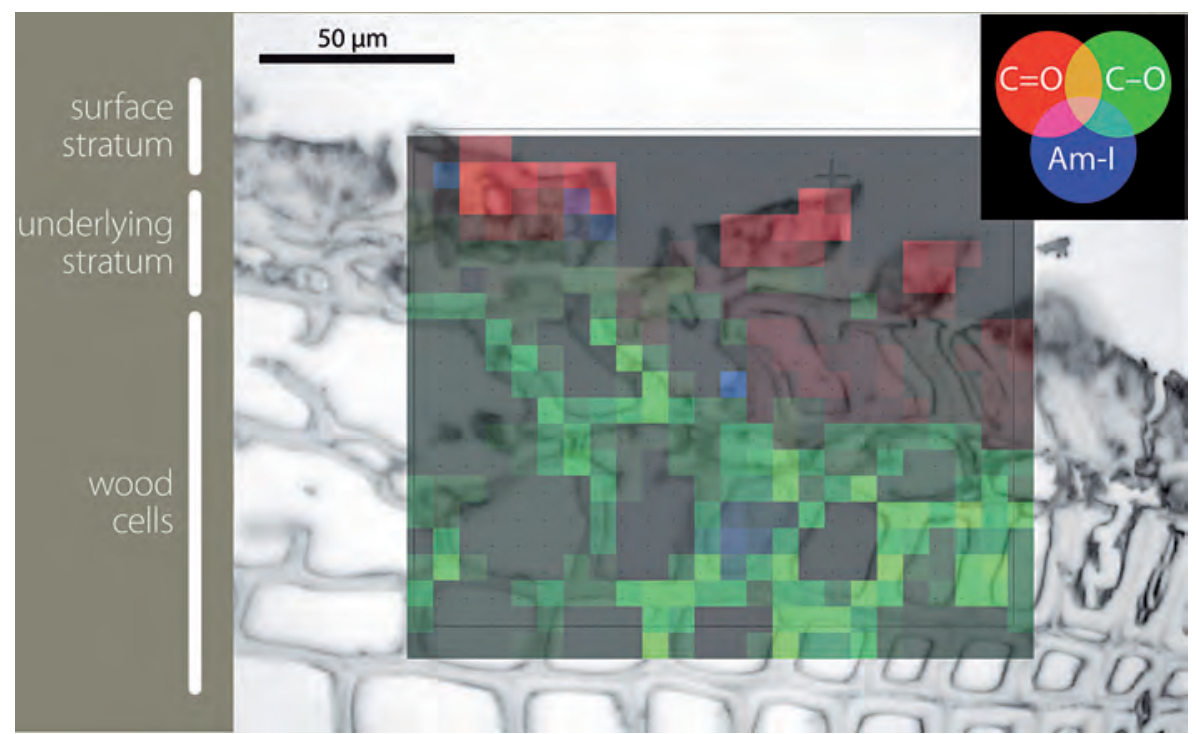

Figure 1. Image de microscopie infrarouge obtenue sur la ligne SMIS du synchrotron SOLEIL sur la coupe du violon le « Provigny » (A. Stradivari, 1716, Crémone, coll. Musée de la musique E. 1730.1). L'image obtenue permet d'identifier et localiser la présence des composés organiques (huile - résine) à l'échelle des cellules du bois et d'exclure la présence de composés protéiques. En vert : liaisons simples $\mathrm{C}-\mathrm{O}$; en rouge : groupements carboxyles; en bleu : amides I, indiquant la présence de protéines - .

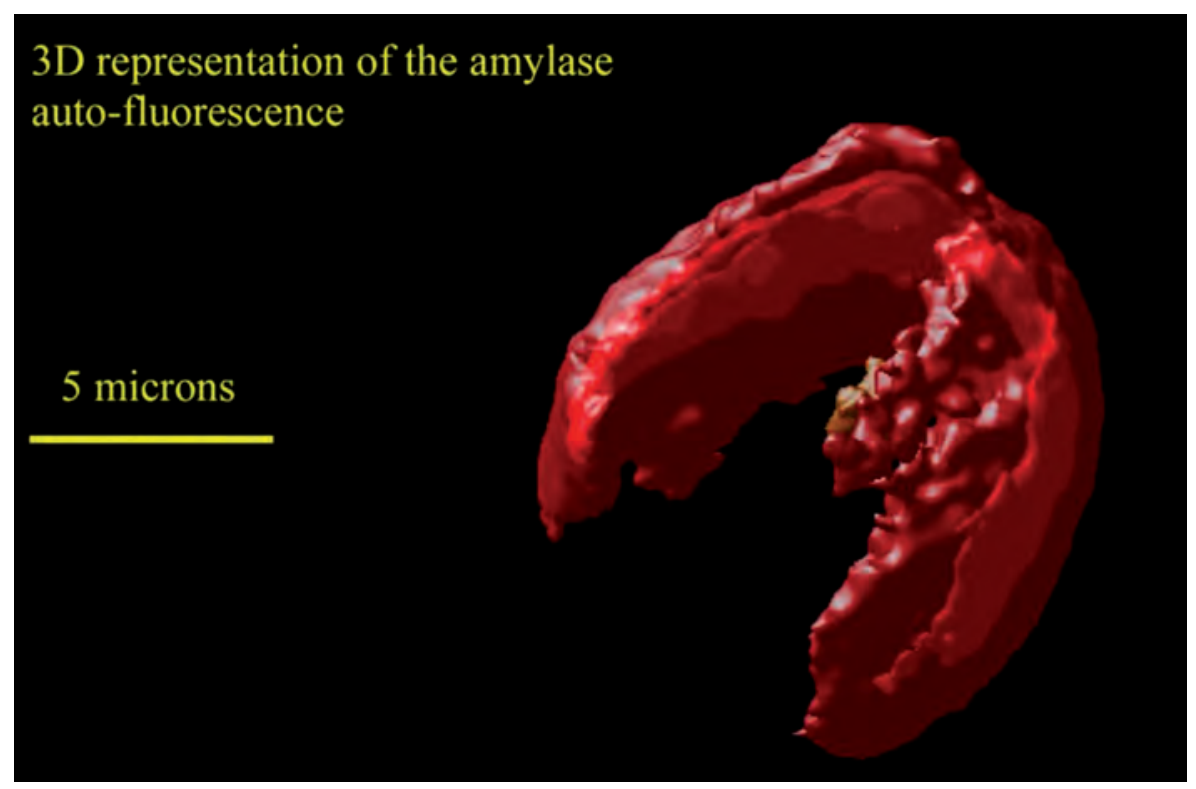

Figure 2. Localisation tridimensionnelle d'une amylase digérant un grain d'amidon. La fluorescence de l'acide aminé tryptophane contenu dans cette enzyme permet de l'imager sans marqueur.

de pin. Stradivarius y a incorporé différents pigments utilisés en peinture (figure 1). Cette étude met donc en évidence l'intention de Stradivarius de donner à ses instruments leurs célèbres teintes rouges.

\section{Les enzymes pour bio-carburants}

Les recherches en chimie verte sont en plein essor. L'intérêt croissant pour les probiotiques (et leur impact sur la santé) et la production de bio carburant motivent de nombreuses études sur la dégradation enzymatique de biopolymères tels que l'amidon et la lignocellulose. L'hydrolyse de ces substrats solides dépend de leurs structures semi-cristallines qui peuvent limiter la diffusion d'enzymes et de ce fait réduire leur dégradation. Une étude

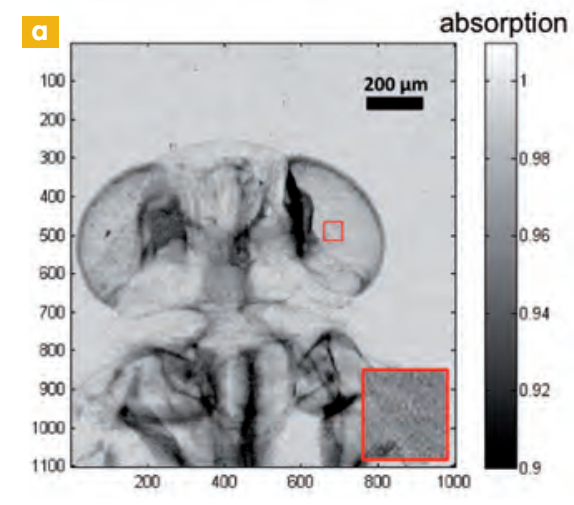

b

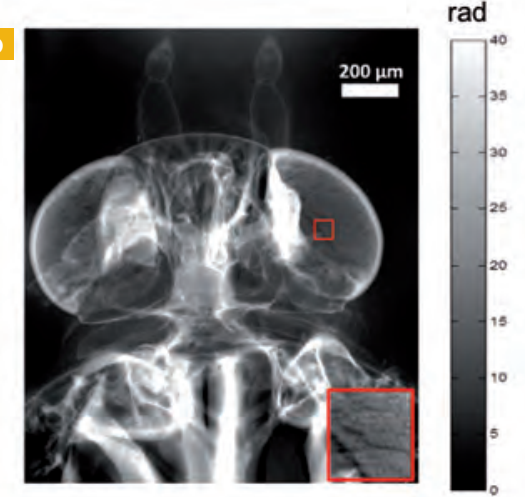

Figure 3. Image d'absorption à $14 \mathrm{keV}$ d'une mite (a). Projection de phase quantitative de la même zone (b) (Adapté de Medjoubi et al.).

réalisée sur la branche «IMAGING » de la ligne de lumière DISCO à SOLEIL a permis de suivre in situ les voies de dégradation de deux enzymes optimisées pour la production de bio-carburant. L'hydrolyse des grains d'amidon a été suivie par autofluorescence du tryptophane (excitation dans la gamme de l'ultraviolet lointain) et a permis d'identifier deux voies de dégradation antagonistes. Le microscope ultraviolet développé sur la ligne DISCO permet de représenter en 3 dimensions l'interaction enzyme-substrat au cours de I'hydrolyse (jusqu'à 10 heures), ce qui en fait un outil particulièrement efficace pour la compréhension et le suivi de l'hydrolyse des substrats solides (figure 2).

\section{Les cellules de l'œil d'une mite}

Les structures cellulaires de l'œil d'une mite sont clairement visibles par contraste de phase X (figure $3 b$ ) tandis que l'image d'absorption simple (figure $3 a$ ) ne permet pas d'obtenir autant de détails. Ce type de contraste est particulièrement approprié aux tissus mous. 


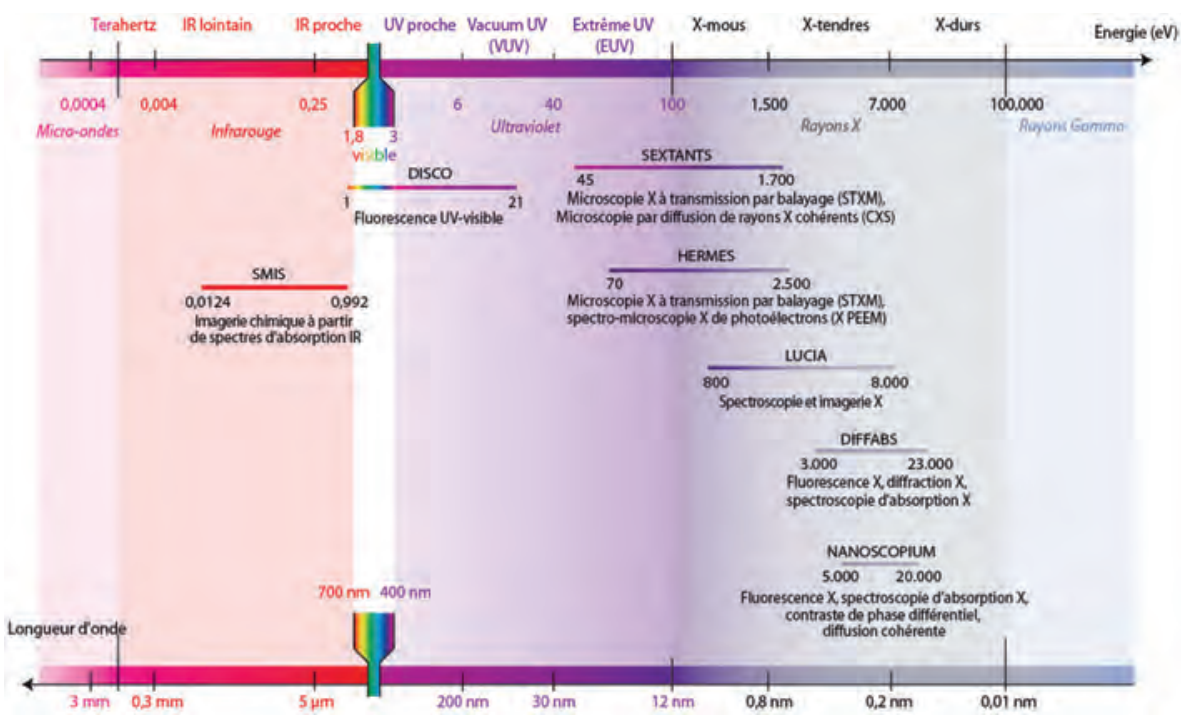

Figure 4. L'ensemble des techniques de microscopie utilisées sur les lignes de SOLEIL.

\section{La corrélation en imagerie}

Naturellement, l'ensemble de ces techniques de microscopies trouve tout son intérêt dans la combinaison entre modalités (figure 4). Le recalage, la superposition et l'intégration des images obtenues sur les différentes lignes de SOLEIL bénéficient d'un travail important. Et, au-delà de corrélations entre lignes, SOLEIL s'est doté de techniques de microscopie qui n'ont pas besoin de rayonnement synchrotron. Un microscope à effet tunnel, un microscope électronique à balayage, un microspectromètre Raman et un microscope multiphotons sont ainsi disponibles pour caractériser les échantillons avant utilisation de la lumière synchrotron.

Enfin, un microscope à force atomique permet de caractériser les optiques nécessaires à chaque technique synchrotron.

En conclusion, grâce à l'utilisation du rayonnement synchrotron de nombreuses techniques de microscopies à rayons $\mathrm{X}$ peuvent être mises en œuvre, avec des performances inégalées par les appareils de laboratoire et guidant leurs développements futurs. De plus, le large spectre en énergie produit par les électrons permet le développement de microscopies complémentaires à ces techniques, dans les domaines de l'infrarouge et de l'ultraviolet lointain.
Afin d'aller plus loin, la combinaison du rayonnement synchrotron avec les techniques de champ proche utilisant une pointe permettra d'obtenir une résolution latérale de l'ordre d'une dizaine de nanomètres en mode balayage de surface (topologie) et une analyse spectrale de I'infrarouge aux UV avec une résolution de quelques nanomètres également, en exploitant des effets de champ proche et de résonances de plasmoniques. Le point clé, sur lequel SOLEIL poursuit une stratégie de développement, reste le couplage optique du rayonnement synchrotron avec une pointe.

\section{Références}

Echard, J.P., Bertrand, L., von Bohlen, A., Le Hô, A.S., Paris, C., Bellot-Gurlet, L., Soulier, B., Lattuati-Derieux, A., Thao, S., Robinet, L., Lavédrine, B., Vaiedelich, S., The nature of the extraordinary finish of Stradivari's instruments, Angewandte Chemie International Edition, 2010, 49(1): 197-201

Jamme, F., Bourquin, D., Tawil, G., Viksø-Nielsen, A., Buleon, A. J., Refregiers, M., 3D imaging of enzymes working in-situ, Analytical Chemistry, 2014, 86(1 1): 5265-5270

Medjoubi, K., Leclercq, N., Langlois, F., Buteau, A., Lé, S., Poirier, S., Mercère, P., Sforna, M.C., Kewish, C.M., Somogyi, A., Development of fast, simultaneous and multi-technique scanning hard $X$-ray microscopy at Synchrotron Soleil, Journal of Synchrotron Radiation, 2013, 20(2): 293-299

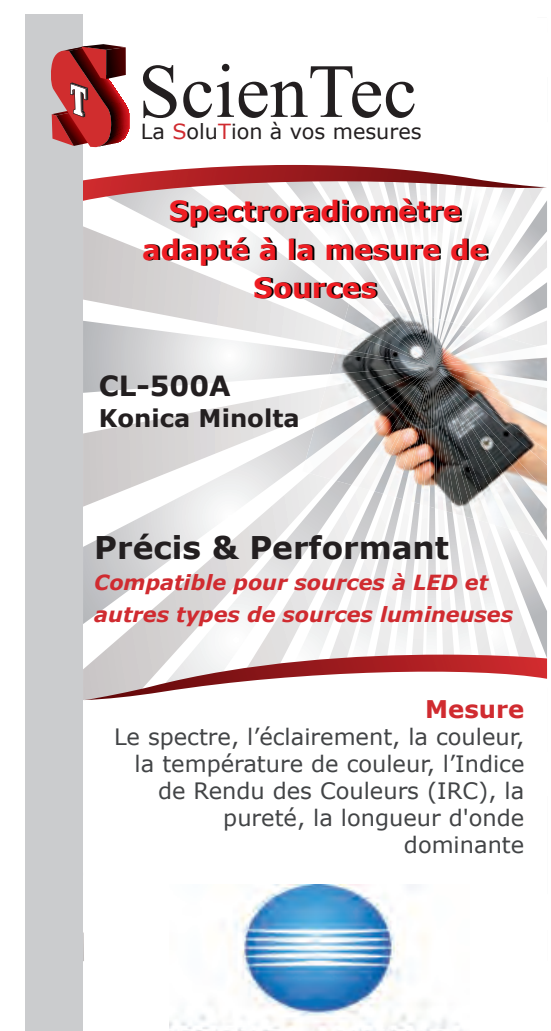

\section{KONICA MINOLTA}

\section{Caractéristiques}

Conforme aux normes DIN et JIS, Performant

Facile à utiliser

Mode autonome ou avec ordinateur

Très bon rapport qualité/prix...

\subsection{0 $x-1>x$ Applications Evaluation des caractéristiques d'émission spectrique \\ Développement, contrôle et maintenance de sources lumineuses $R \& D$ pour les éclairages Réalisation et ajustement d'ambiances lumineuses Maintenance des cabines lumineuses}

SCIENTEC, c'est aussi :

Spectroradiomètres Vidéo-colorimètres Photomètres

Luxmètres

Chromamètres

Sources de Référence 01.64.53.27.00 